\title{
Peritectic Phase Transition of Benzene and Acetonitrile and Formation of a Cocrystal Relevant to Titan, Saturn's Icy Moon
}

\author{
Christina A. McConville, ${ }^{a}$ Yunwen Tao, ${ }^{a}$ Hayden A. Evans, ${ }^{b}$ Benjamin A. Trump, ${ }^{b}$ Jonathan B. Lefton, ${ }^{a}$ Wenqian Xu, ${ }^{c}$ Andrey A. \\ Yakovenko, ${ }^{\mathrm{c}}$ Elfi Kraka, ${ }^{\mathrm{a}}$ Craig M. Brown ${ }^{\mathrm{b}, \mathrm{d}}$ and Tomče Runčevski*a \\ a. Department of Chemistry, Southern Methodist University, Dallas, TX 75275 (USA) E-mail: truncevski@smu.edu \\ b. National Institute of Standards and Technology, Center for Neutron Research, Gaithersburg, MD 20899 (USA) \\ c. X-ray Science Division, Advanced Photon Source, Argonne National Laboratory, Argonne, IL 60439 (USA) \\ d. Department of Chemical and Biomolecular Engineering, University of Delaware, DE 19716 (USA)
}

Benzene and acetonitrile are two of the most commonly used solvents found in almost every chemical laboratory. Titan, Saturn's icy moon, is one other place in the Solar system that has even larger amounts of these compounds, together with many other hydrocarbons. On Titan, organic molecules are produced in the atmosphere and carried by methane rainfall to the surface, where they either dissolve in the lakes, deposit as sandy dunes, or solidify as minerals with complex composition and structure. In order to untangle these structural complexities a reliable model of the phase behavior of these compounds at temperatures relevant to Titan is crucial. We therefore report the composition-temperature binary phase diagram of acetonitrile and benzene, and provide a detailed account of the structure and composition of the phases. This work is based on differential scanning calorimetry and in situ powder diffraction analyses with synchrotron X-ray radiation and supported by theoretical modeling. Benzene and acetonitrile were found to undergo a peritectic reaction into a cocrystal with a 1:3 acetonitrile:benzene stoichiometry. The crystal structure was solved and refined in the polar space group, $R 3$, and the solution was confirmed and optimized by energy minimization calculations. To mimic the environment on Titan more accurately, we tested the stability of the structure under liquid ethane. The diffraction data indicate that the cocrystal undergoes further change upon contact with ethane. These results provide new insights into the structure and stability of a potential mineral on Titan, and contribute to the fundamental knowledge of some of the smallest organic molecules.

\section{Introduction}

Benzene and acetonitrile are some of the most commonly used solvents in the chemical industry, with research on benzene ${ }^{1 \mathrm{a}}$ and acetonitrile $^{1 \mathrm{~b}}$ dating back to the earliest days of modern chemistry. For example, the determination of the crystal structure of hexamethylbenzene ${ }^{2 a}$ in 1928 was one of the earliest milestones in crystallography, with reports on the crystal structures of pure benzene and acetonitrile following in $1958^{2 \mathrm{~b}, \mathrm{c}}$ and 1981 , $^{2 \mathrm{~d}, \mathrm{e}}$ respectively. The phase diagram of these compounds was first studied in $1934,{ }^{3 a}$ and later revisited in $1961 .^{3 b}$ These early and incomplete studies suggest the formation of a cocrystal with unknown stoichiometry. To the best of our knowledge, the structure of the cocrystal, as well as the nature of the molecular interactions of acetonitrile and benzene in its solid state, remain unknown. In fact, due to limited practical applications, cryogenic research on solvents and room-temperature liquids is relatively scarce. However, this research field has now become increasingly relevant with the advances in space exploration and planetary science, where these molecules play a crucial role.

Last year, NASA initiated a monumental step in space exploration with their announcement of the New Frontiers mission to Titan, Saturn's icy moon. ${ }^{4}$ A rotorcraft, aptly named Dragonfly, will launch in 2026 and is expected to arrive on the surface of Titan in 2034. This endeavour is timely preceded by the Cassini-Huygens mission (1997-2017), which was critical to the characterization of Saturn and its moons. ${ }^{5}$ The Cassini-Huygens mission confirmed that Titan possesses a dense and chemically active atmosphere composed mainly of dinitrogen and methane. Fuelled by radiation from the Sun and Saturn's magnetosphere, dinitrogen and methane react to produce various organic molecules, ${ }^{6}$ including benzene and acetonitrile, which are then carried by methane rainfall and vigorous storms $^{7}$ to the surface, where they dissolve in the hydrocarbon lakes. ${ }^{8}$ A seasonal cycling of evaporation and precipitation, a process notably similar to Earth's hydrological cycle, produces evaporite lakebeds and mineral deposits made of organic molecules. ${ }^{9}$ During its 2.7 -year baseline mission, Dragonfly will explore Titan's lakebeds, dunes and the floor of an impact crater, searching for signatures of extinct, extant, or future life. ${ }^{4}$ Laboratory studies directed towards predicting, modelling and recreating the surface mineralogy of the moon will greatly aid to this mission.

Benzene and acetonitrile are expected to be produced in the atmosphere of Titan by radical and ion facilitated reactions. ${ }^{10}$ One theoretical model estimates constant precipitation fluxes of $1.08 \mathrm{x}$ $10^{6}$ and $1.27 \times 10^{7}$ molecules $/ \mathrm{cm}^{2} / \mathrm{s}$ for benzene and acetonitrile, respectively, among other organic compounds. ${ }^{10 a}$ If we consider a scenario in which the precipitation cycle repeats for over 4 billion years (the age of Titan), the accumulated surface layer of aromatics could to reach up to 3 meters. ${ }^{10 \mathrm{~b}}$ Under the surface conditions, 93.6 $\mathrm{K}$ and $1.4 \mathrm{~atm}$, molecular benzene and acetonitrile may exist as dissolved species in the ethane-rich lakes or as minerals in the solid state. These organic minerals can exist as single-phase molecular crystals, ${ }^{2}$ but also as cocrystals, solvates, hydrates and clathrates. Recently, a cocrystal of benzene and ethane, was thoroughly studied and characterized as a potential mineral on Titan. ${ }^{11}$ Several other cocrystals relevant to Titan have recently been considered, including acetylene and ammonia, ${ }^{12 \mathrm{a}}$ acetylene and butane, ${ }^{12 \mathrm{~b}}$ and a ternary phase cocrystal of benzene, acetylene and hydrogen cyanide, ${ }^{12 \mathrm{c}}$ complementing previous studies of small-molecule binary phases, such as the cocrystal of acetylene and benzene. ${ }^{12 \mathrm{~d}}$

Herein, we report the composition-temperature $(\chi-T)$ phase diagram of benzene and acetonitrile, constructed using differential 
scanning calorimetry (DSC) and in situ powder X-ray diffraction (PXRD) with synchrotron radiation. We observe a reaction between solid benzene and liquid acetonitrile with a well-defined peritectic line. The crystal structure of the peritectic product was solved from powder diffraction data and optimized with density functional theory (DFT) methods. It was revealed that the phase exists as a 1:3 acetonitrile:benzene cocrystal. The thermal expansion properties and stability of the cocrystal were studied by calculation of the principle axes of expansion. Finally, we studied the behaviour of the cocrystal immersed in liquid ethane, in order to mimic the environmental conditions expected in/around the lakes of Titan.

\section{Results and Discussion}

\section{Temperature-composition phase diagram}

The early reports on the $\chi-T$ phase diagram of benzene and acetonitrile were based on a visual observation of the melting point variations as a function of composition. ${ }^{3}$ In order to provide a more accurate description, we revisited the system using DSC as a modern thermoanalytical technique (more information provided in the ESI). Considering the sensitivity of crystallization events to the experimental conditions (supercooling, presence of impurities, etc.), the binary phase diagram was constructed using the endothermic peak maxima of the heat flux in the DSC curves collected upon heating (Figure 1a). Figure $1 \mathrm{~b}$ presents the $\chi-T$ binary phase diagram for the acetonitrile-benzene system, expressed as function of the mole fraction of acetonitrile, $\chi\left(\mathrm{CH}_{3} \mathrm{CN}\right)$. The presence of a secondary phase decreased the melting points of both pure benzene $\left(\boldsymbol{T}_{\mathbf{B}}=279 \mathrm{~K}\right)$ and acetonitrile $\left(\boldsymbol{T}_{\mathbf{A}}=230 \mathrm{~K}\right)$. The gradual decrease clearly outlines an upper fusion boundary line (f) converging to a eutectic halt $(\mathbf{E})$ at $T=224 \mathrm{~K}$ and $\chi\left(\mathrm{CH}_{3} \mathrm{CN}\right)=0.9$. The upper fusion line, the eutectic line and the eutectic point are all in good agreement with previously reported data $\left(\mathbf{E}=224.45^{3 \mathrm{a}}\right.$ and $\left.224.82^{3 \mathrm{~b}} \mathrm{~K}\right)$. A comparison with the earlier binary phase diagrams is given in Figure $\mathrm{S} 2$ in the ESI. A peritectic reaction between solid benzene, $s-\mathrm{C}_{6} \mathrm{H}_{6}$, and liquid acetonitrile, $l-\mathrm{CH}_{3} \mathrm{CN}$, was observed at $248 \mathrm{~K}$, establishing a peritectic ${ }^{\#}$ boundary line (p) extending to $\chi\left(\mathrm{CH}_{3} \mathrm{CN}\right)$ $=0.55$. The DSC measurements revealed a previously unobserved region in the binary phase diagram, which is indicative of the phase change of acetonitrile from the low-temperature $\beta$ form to the hightemperature $\alpha$ form. ${ }^{2 \mathrm{e}}$ This phase transition was detected as a series of relatively weak endothermic events outlining a solid-to-solid transition (t) line at $217 \mathrm{~K}$. The overall shape of the $\chi-T$ binary phase diagram of benzene and acetonitrile is typical for a solid-liquid binary phase diagram of two components featuring incongruent melting.

\section{Structural description}

The calorimetric study of the $\chi-T$ binary phase diagram clearly outlined a region of incongruent melting and a peritectic phase transition, however, it did not provide structural information of the constituent phases. To investigate the solid state structures and composition in the regions below both the fusion and peritectic lines, we performed variable-temperature in situ PXRD measurements. Figure 2a presents the PXRD data of a mixture of acetonitrile and benzene in a 1:3 ratio, shown as a 2D plot of scattered intensity as a function of temperature. A Rietveld refinement ${ }^{13}$ analysis indicated that above the peritectic line, from 263 to $245 \mathrm{~K}$, solid benzene (in the Pbca


Fig. 1 a) DSC curves of mixtures of benzene and acetonitrile, prepared as varying mole fraction of acetonitrile collected upon heating of the samples. b) Binary composition-temperature phase diagram of benzene and acetonitrile, constructed by plotting the endothermic events observed in the DSC curves collected upon heating. The composition of the condensed phase regions was determined by X-ray diffraction analyses. 
polymorphic modification) is the only crystalline phase present in the system (Figure 2b). Below $245 \mathrm{~K}$, additional reflections were observed in the measured patterns, corresponding to the formation of a secondary crystalline phase. The PXRD pattern collected at 100 $\mathrm{K}$ was used to solve and refine the crystal structure of this phase (further details are provided in the ESI). The unit cell symmetry size indicated that the stoichiometry of the cocrystal is $1: 3$ acetonitrile:benzene, $\chi\left(\mathrm{CH}_{3} \mathrm{CN}\right)=0.25$. The crystal structure was solved in the trigonal space group $R 3$ and was later confirmed and refined by the Rietveld method ${ }^{13}$ (Figure $2 c$ ).

The crystal packing diagram of the cocrystal is presented in Figure 3a with selected structural and crystallographic information in Table S1 . The structure is related to the structure of benzene ${ }^{2 b, c}$ and the 1:3 ethane:benzene cocrystal (Figure S3). ${ }^{11 \mathrm{c}}$ The packing features channels of benzene molecules, self-assembled along a $\overline{3}$ axis parallel to the $c$ crystallographic direction. The channels are stabilized by weak $\mathrm{C}-\mathrm{H} \cdots \pi$ interactions. Acetonitrile molecules positioned along the channels with a relatively short $\mathrm{CH}_{3} \mathrm{CN} \cdots \mathrm{CH}_{3} \mathrm{CN}$ distance of $3.18(1) \AA$. The ordered, linear arrangement or the asymmetric acetonitrile molecules reduces the symmetry of the crystal structure from the space group $R \overline{3}$ to $R 3$. It should be noted that the crystal structure can also be described in the $R \overline{3}$ space group, with disordered acetonitrile molecules in the crystal packing. Due to intrinsic limitations of the powder diffraction method, the $R \overline{3}$ and $R 3$ space groups cannot be distinguished based on the observed absences of reflections.
To validate the crystal packing, and to assess the possibility for a disordered structure, we performed energy optimization (further technical details are provided in the ESI), on isolated $\left(\mathrm{CH}_{3} \mathrm{CN}\right)_{2}\left(\mathrm{C}_{6} \mathrm{H}_{6}\right)_{12}$ clusters. In one of the clusters, the starting relative atomic positions were taken as observed by the crystallographic analysis, representing the ordered, $R 3$ structure. In the other cluster, the two $\mathrm{CH}_{3} \mathrm{CN}$ molecules were related by a center of inversion. Upon relaxation of the atomic position, only the noncentrosymmetric cluster (Figure $3 b$ ) reached a local minimum on the potential energy surface, as confirmed by vibrational analysis. The centrosymmetric cluster was shown to be significantly less stable ( $6 \mathrm{kcal} / \mathrm{mol}$ ). These calculations support the model of the crystal structure in the $R 3$ polar space group and the optimized structure was found to be in excellent agreement with the experimentally obtained crystal structure (see an overlay Figure S4).

Crystallization in a polar space group may have important implications on the physical properties. Acetonitrile is a highly polar molecule with a dipole moment of $\mu=13.17 \times 10^{-30} \mathrm{Cm}$. The alignment of polar molecules onto a 3-fold axis, in the absence of a center of inversion, gives a polar macroscopic crystal. Polar crystals are functional materials that can exhibit pyroelectric, piezoelectric, ferroelectric and/or electrooptic effects, as well as second harmonic generation, triboluminescence and other useful properties. ${ }^{14}$ The melting point below room temperature limits potential applications of $s-\left(\mathrm{CH}_{3} \mathrm{CN}\right)\left(\mathrm{C}_{6} \mathrm{H}_{6}\right)_{3}$ in most current technologies, but because this material is stable under the conditions on Titan, it can potentially be used as an integral part of the design of on-board instruments for
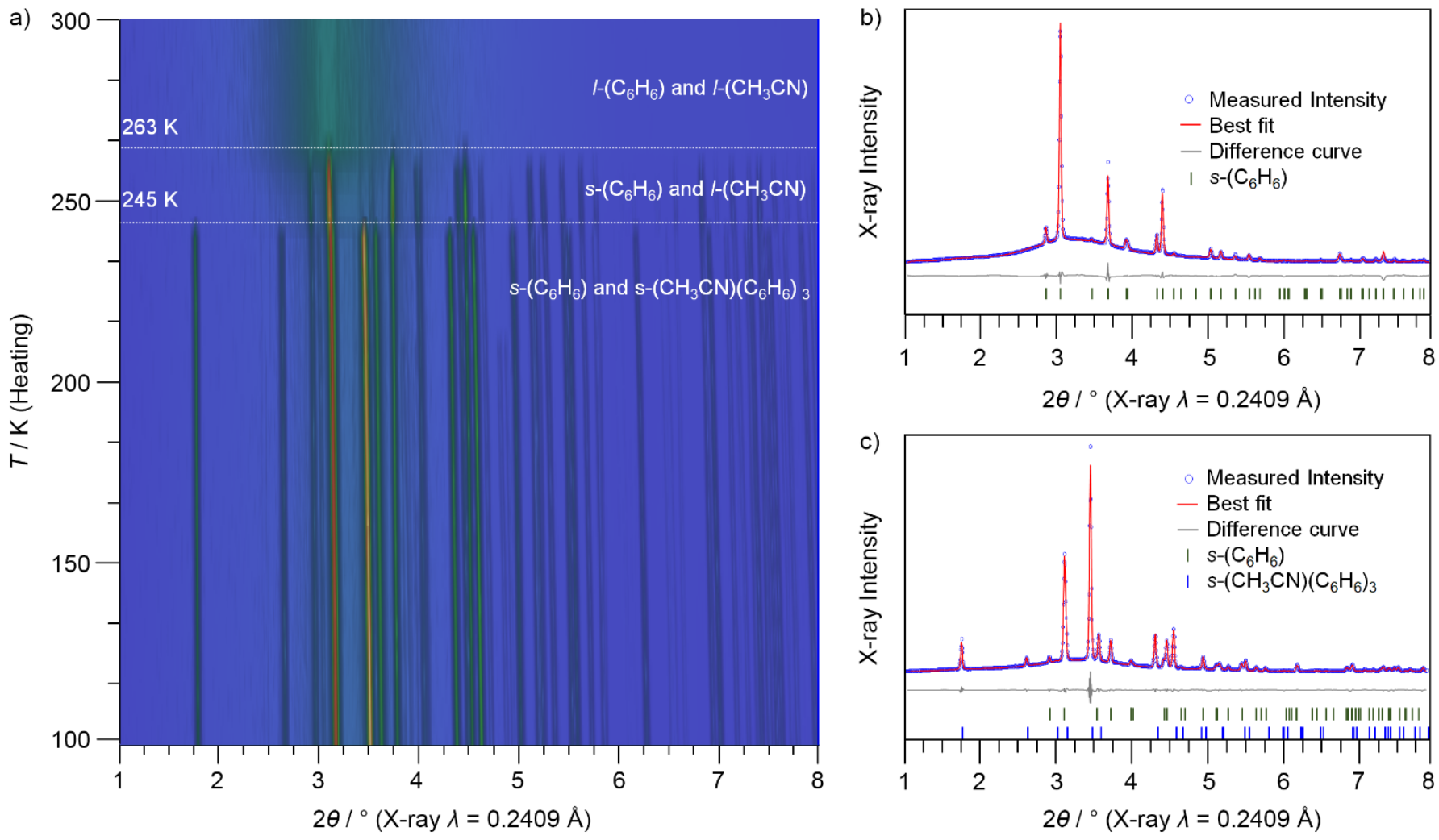

Fig. 2 a) Variable-temperature PXRD data collected upon heating of sample of acetonitrile and benzene prepared in 1 to 3 ratio. Representative Rietveld refinement plots of the patterns collected at $251 \mathrm{~K}$ (single phase refinement) and $100 \mathrm{~K}$ (double phase refinement) are presented in b) and c), respectively. 
missions such as Dragonfly. Finally, it should be mentioned that with these crystal-packing features, the acetonitrile:benzene 1:3 cocrystal can serve as a potential precursor in pressure-induced polymerization reactions toward functionalized graphene structure, ${ }^{15 \mathrm{a}}$ and nitrogen-rich compounds, ${ }^{15 \mathrm{~b}}$ which is relevent for developing alternative solid-state synthetic routes.

The similarity of the crystal structure of the 1:3 acetonitrile:benzene cocrystal and the $\mathrm{Pbca}$ polymorphic modification of crystalline benzene explains the tendency of solid benzene to react with liquid acetonitrile and form a peritectic product. Figure $3 \mathrm{c}$ shows the crystal packing before and after the peritectic phase transition, which can be envisaged as a replacement of a benzene molecule for acetonitrile, and subsequent reorganization of the lattice of benzene molecules while maintaining the network of close $\mathrm{C}-\mathrm{H} \cdots \pi$ interactions.

\section{Thermal expansion and stability}

The in situ diffraction data were used to assess thermal stability of the cocrystal. Crystallographic analyses of the data collected upon cooling and heating of 1:3 mixture of acetonitrile and benzene indicate that the cocrystal forms upon cooling at $227 \mathrm{~K}$ (crystalizing in a phase mixture with benzene) and it is stable upon further decrease of the temperature (Figure S7). Upon heating, the cocrystal is stable up to a melting temperature of $245 \mathrm{~K}$ (Figure S8).

Molecular solids respond to a decrease in temperature with decreases in atomic thermal vibrations, resulting in positive linear thermal expansion. ${ }^{15 a}$ The thermal expansion of the $1: 3$ acetonitrile:benzene cocrystal was studied by observing the changes of the unit cell parameters (further details are provided in the ESI). The principal axes ${ }^{\S}$ of linear expansion were calculated as orthogonal strains based on the variation of the unit cell parameters. ${ }^{15 \mathrm{~b}, \mathrm{c}}$ The $x_{1}$ and $x_{2}$ principal axes were found to coincide with the $c$ and $a$ crystallographic axes, respectively. The third principal axis, $x_{3}$, was found to be approximately parallel to the [210] crystal axis, which aligns with the direction of the short benzenebenzene interactions between neighbouring benzene rings in the crystal packing. The axial linear thermal expansion coefficients, $a_{1}$ $=95(3) \times 10^{-6} \mathrm{~K}^{-1}$ and $a_{2,3}=131(3) \times 10^{-6} \mathrm{~K}^{-1}$, result in a volumetric expansion coefficient of $366(9) \times 10^{-6} \mathrm{~K}^{-1}$. These values are significantly higher than the typical expansion coefficients of molecular solids $\left(<20 \times 10^{-6} \mathrm{~K}^{-1}\right) .{ }^{15 a}$ This thermal expansion is a consequence of the crystal packing being based exclusively on weak supramolecular interactions (such as van der Waals forces), which are particularly sensitive to atomic thermal vibrations.

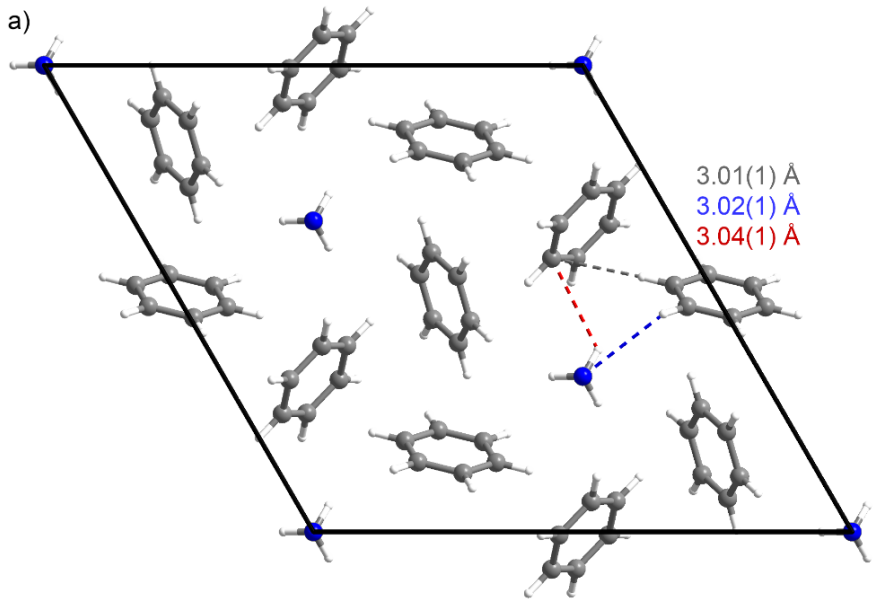

$1: 3$ acetonitrile:benzene, $R 3$

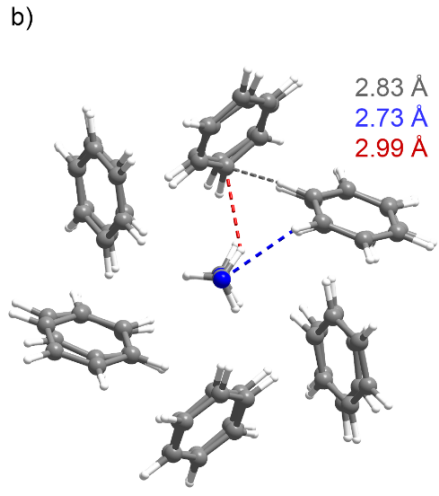

(acetonitrile) $)_{2}(\text { benzene })_{12}$ cluster

c)
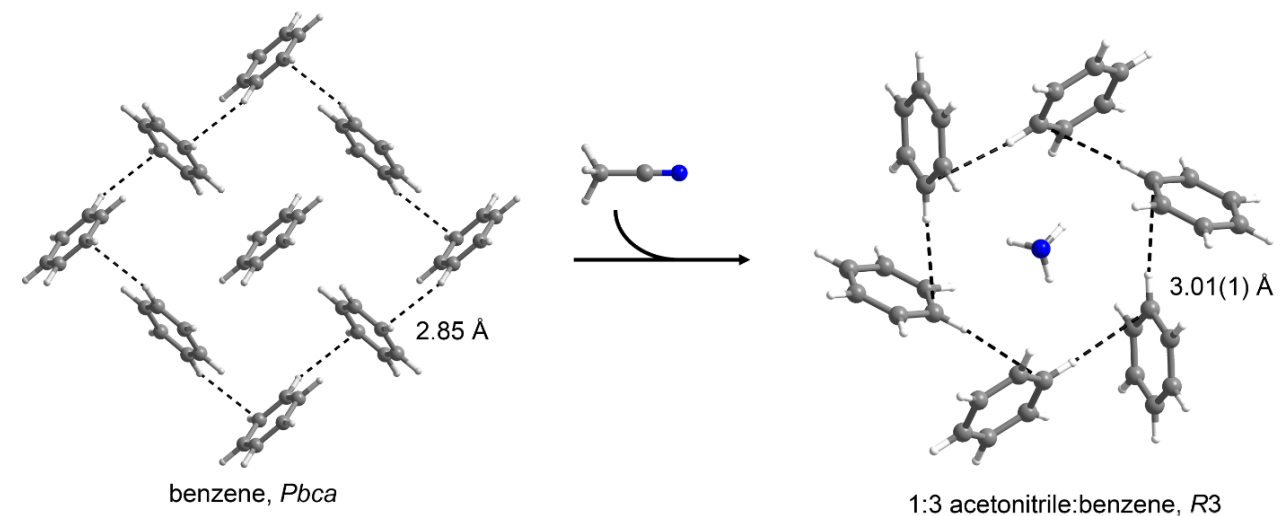

$1: 3$ acetonitrile:benzene, $R 3$

Fig. 3 a) Crystal packing diagram of the 1:3 acetonitrile:benzene cocrystal. b) Representation of the optimized cluster by energy minimization. c) Fragments of the crystal packing diagrams of benzene and the cocrystal schematically representing the peritectic phase transition. Legend: nitrogen in blue, carbon in grey, hydrogen in white. 


\section{Reaction with ethane}

In order to test the stability of the 1:3 acetonitrile:benzene cocrystal under conditions relevant to evaporite deposits on Titan, we performed in situ analyses of the cocrystal upon contact with liquid ethane. Acetonitrile and ethane have the same polar molecular diameter, and can fit within the channels formed by benzene molecules (Figure S3). In fact, the only structural difference between these cocrystals is in their symmetry; the ethane:benzene cocrystal forms in the space group $R \overline{3}$, while acetonitrile:benzene forms in a space group of lower symmetry, $R 3$, as a result of the asymmetry of the acetonitrile molecule.

To explore this ternary system, we filled a capillary with a 1:3 mixture of acetonitrile and benzene and we saturated the atmosphere in the capillary with gaseous ethane. After that, we slowly decreased the temperature to $100 \mathrm{~K}$ and collected diffraction data (Figure 4). Diffraction data were also collected upon heating at $200 \mathrm{~K}$ and 240 $\mathrm{K}$. Interestingly, the diffraction pattern does not correspond to either of the cocrystals in a phase-pure form. Further experiments and analyses are necessary to solve the crystal structure of this phase or potential mixture of phases. This experimental observation hints at the possibility of mixed - and potentially non-stoichiometriccrystalline phases, which would have a significant impact on the approach to identifying potential minerals on Titan. This possibility draws yet another parallel between the mineralogy on Titan and the mineralogy on Earth, where non-stoichiometric phases frequently occur. Furthermore, these results indicate that the 1:3 acetonitrile:benzene cocrystal may be used as an indicator for the past presence of ethane. This type of structural markers may become relevant for on-site analyses of the Titanean surface terrain by the Dragonfly rotorcraft.

\section{Conclusions}

The recently concluded Cassini-Huygens mission to Titan and the forthcoming Dragonfly mission have reignited interest in the fundamental organic chemistry of small molecules. Here, we report the $\chi-T$ phase diagram of benzene and acetonitrile, which features incongruent melting and a peritectic phase transition of solid benzene and liquid acetonitrile into a 1:3 acetonitrile:benzene cocrystal. The crystal $\&$ structure of the cocrystal was solved and refined from in situ $\$$ diffraction data collected using synchrotron radiation. The system crystalized in the polar space group $R 3$ and is characterized by a 1 trigonal structure of benzene molecules self-assembled into hexagonal channels; these channels surround the acetonitrile ${ }^{2}$ molecules, which are in a parallel alignment with the central 3-fold axis. Under conditions relevant to the surface around the lakes of Titan, benzene and acetonitrile react with ethane and produce complicated mixture of phases. These results highlight the pronounced structural and compositional diversity of potential ${ }^{3}$ minerals on Titan.

\section{Conflicts of interests}

There are no conflicts to declare.

\section{Acknowledgements}

T.R. and C.A.M. thank the Robert A. Welch Foundation (Grant No.: N-2012-20190330) for financial support. C.A.M. thanks the Texas

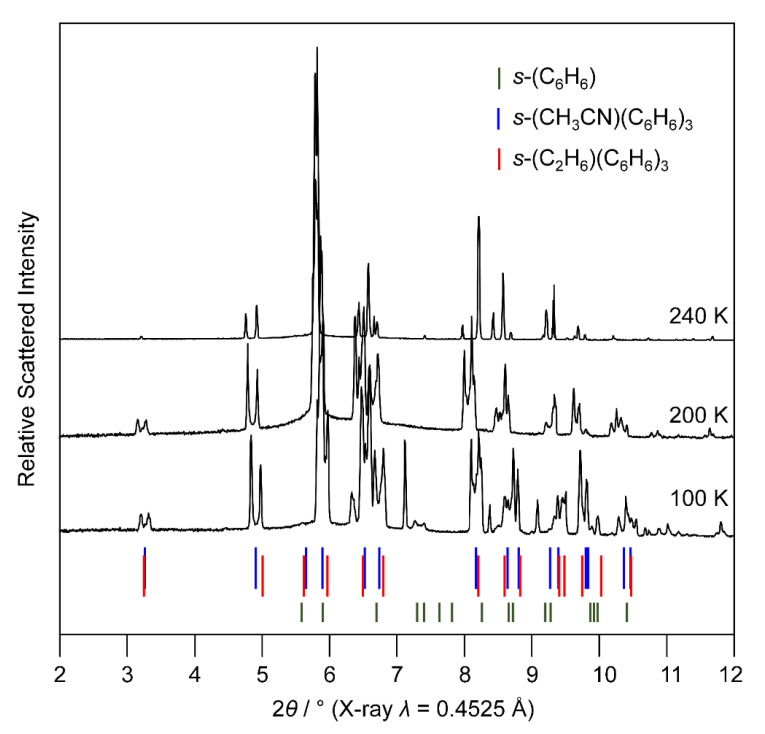

Fig. 4 Powder diffraction patterns of a system made of acetonitrile and benzene in a 1:3 ratio, crystallized in a environment saturated with liquid ethane.

Space Grant Consortium graduate fellowship. H.A.E. thanks the National Research Council (USA) for financial support through the Research Associate Program. We thank SMU for providing generous computational resources. This research used Beamline 17$\mathrm{BM}$ at the Advanced Photon Source, a U.S. Department of Energy (DOE) Office of Science User Facility operated for the DOE Office of Science by Argonne National Laboratory under Contract No. DEAC02-06CH11357.

\section{Notes and references}

+ CCDC, The Cambridge Crystallographic Data Centre

In the early literature, peritectic transitions are sometimes referred to as meritectic transitions.

Due to the trigonal symmetry (space group $R 3$ ) of the unit cell of $1: 3$ acetonitrile:benzene cocrystal, the crystallographic axes do not coincide with the principal axes of linear expansion.

a) H. E. Armstrong, Nature, 1925, 115, 1010-1013; b) J.-B. Dumas, Comptes rendus., 1847, 25, 383-384.

a) K. Lonsdale, Nature, 1928, 122, 810; b) E. Cox, D. Cruickshank and J. Smith, Proc. R. Soc. A: Math. Phys. Engineering Sci., 1958, 247, 1-21; c) G. E. Bacon, N. A. Curry and S. A. Wilson, Proc. R. Soc. A: Math. Phys. Engineering Sci. 1964, 279, 98-110; d) M. J. Barrow, Acta Cryst. B, 1981, 37, 2239-2242; e) O. K. Antson, K. J. Till and N. H. Andersen, Acta Cryst. B, 1987, 43, 296-301.

a) N. I. Joukovsky, Bull. Soc. Chim. Belges., 1934, 43, 397-446; b) J. R. Goates, J. B. Ott and A. H. Budge, J. Phys. Chem., 1961, 21622165.

4 "NASA's Dragonfly Will Fly Around Titan Looking for Origins, Signs of Life" NASA Press Release 19-052, June 27, 2019.

5 a) Titan from Cassini-Huygens, R. H. Brown, J-P. Lebreton, J. H. Waite, Springer, Heidelberg, 2009; b) H. B. Niemann, S. K. Atreya, S. J. Bauer, G. R. Carignan, J. E. Demick, R. L. Frost, D. Gautier, J. A. Haberman, D. N. Harpold, D. M. Hunten, G. Israel, J. I. Lunine, W. T. Kasprzak, T. C. Owen, M. Paulkovich, F. Raulin, E. Raaen and S. H. Way, Nature, 2005, 438, 779-784; c) A. Coustenis and M. Hirtzig, Res. Astron. Astrophys., 2009, 9, 249-268. 
6 a) S. Gupta, E. Ochiai and C. Ponnamperuma, Nature 1981, 293, $752-$ 727; b) R. D. Lorenz, C. P. McKay and J. I. Lunin, Science, 1997, 275, 642-644.

7 a) C. A. Griffith, J. L. Hall and T. R. Geballe, Science, 2000, 290, 509513; b) R. Hueso and A. Sánchez-Lavega, Nature, 2006, 442, 428431; c) E. L. Schaller, H. G. Roe, T. Schneider and M. E. Brown, Nature, 2009, 460, 873-875.

8 E. R. Stofan, C. Elachi, J. I. Lunine, R. D. Lorenz, B. Stiles, K. L. Mitchell, S. Ostro, L. Soderblom, C. Wood, H. Zebker, S. Wall, M. Janssen, R. Kirk, R. Lopes, F. Paganelli, J. Radebaugh, L. Wye, Y. Anderson, M. Allison, R. Boehmer, P. Callahan, P. Encrenaz, E. Flamini, G. Francescetti, Y. Gim, G. Hamilton, S. Hensley, W. T. K. Johnson, K. Kelleher, D. Muhleman, P. Paillou, G. Picardi, F. Posa, L. Roth, R. Seu, S. Shaffer, S. Vetrella and R. West, Nature, 2007, 445, 61-64.

9 a) D. Cordier, T. Cornet, J. W. Barnes, S. M. MacKenzie, T. Le Bahers, D. Nna-Mvondo, P. Rannou and A. G. Ferreira, Icarus, 2016, 270, 41-56; b) H. E. Maynard-Casely, M. L. Cable, M. J. Malaska, T. H. Vu, M. Choukroun and R. Hodyss, Am. Mineral., 2018, 103, 343349.

10 a) V. A. Krasnopolsky, Icarus 2009, 201, 226-256; b) V. Vuitton, R. V. Yelle and J. Cui, J. Geo. Res. Planets, 2008, 113, E05007; c) G. J. Molina-Cuberos, K. Schwingenschuh, J. J. López-Moreno, R. Rodrigo, L. M. Lara and V. Anicich, J. Geo. Res. Planets, 2002, 107, 9-1-9-11.

11 a) T. H. Vu, M. L. Cable, M. Choukroun, R. Hodyss and P. Beauchamp, J. Phys. Chem., 2014, 118, 4087-4094; b) M. L. Cable, T. H. Vu, R. Hodyss, M. Choukroun, M. J. Malaska and P. Beauchamp, Geophys. Res. Lett., 2014, 41, 5396-5401; c) H. E. Maynard-Casely, R. Hodyss, M. L. Cable, T. Hoang Vu and M. Rahm, IUCrJ, 2016, 3, 192-199.

12 a) M. L. Cable, T. H. Vu, H. E. Maynard-Casely, M. Choukroun and R. Hodyss, ACS Earth Space Chem., 2018, 2, 366-375; b) M. L. Cable, H. T. Vu, M. J. Malaska, H. E. Maynard-Casely, M. Choukroun and Robert Hodyss, ACS Earth Space Chem., 2019, 3, 2808-2815; c) C. Ennis, M. L. Cable, R. Hodyss and R. H. E. Maynard-Casely, ACS Earth Space. Chem., 2020 doi:10.1021/acsearthspacechem.0c00130; d) R. Boese, T. Clark and A Gavezzotti, Helv. Chim. Acta, 2003, 86, 1085-1100.

13 H. M. Rietveld, J. Appl. Crystallogr., 1969, 2, 65-71.

14 a) D. Y. Curtin and I. C. Paul, Chem. Rev., 1981, 81, 525-541; b) K. T. Holman, A. M. Pivovar and M. D. Ward, Science, 2001, 294, $1907-$ 1911.

15 a) Y. Wang, X. Dong, X. Tang, H. Zheng, K. Li, X. Lin, L. Fang, G. Sun, X. Chen, L. Xie, C. L. Bull, N. P. Funnell, T. Hattori, A. SanoFurukawa, J. Chen, D. K. Hensley, G. D. Cody, Y. Ren, H. Hwi Lee and H.-k. Mao, Angew. Chem. Int. Ed. 2019, 58, 1468-1473; b) A. Olejniczak and A. Katrusiak, J. Phys. Chem. B, 2008, 112, 7183-7190.

16 a) Krishnan, R. S., Srinivasan, R. \& Devanarayanan, S. Thermal Expansion of Crystals (Pergamon, 1979); b) Nye, J.F. (1957). Physical Properties of Crystals (Oxford University Press, Oxford); c) M. J. Cliffe and A. L. Goodwin, J. Appl. Cryst., 2012, 45, 1321-1329. 